\title{
Activités de pâturage, biodiversité et végétation pastorale dans la zone Ouest du Burkina Faso
}

\author{
E. Botoni/Liehoun ${ }^{1 *}$ P. Daget ${ }^{2}$ J. César ${ }^{3}$
}

\begin{abstract}
Mots-clés
Bovin - Biodiversité - Végétation Savane - Detarium microcarpum Dynamique des populations Pâturage - Burkina Faso.
\end{abstract}

\begin{abstract}
Résumé
L'évolution de la biodiversité végétale en fonction de trois niveaux de pression foncière et pastorale (faible, modérée, forte) a été étudiée sur des parcours communautaires de la région subhumide du Burkina Faso. L'étude a été conduite sur des faciès de savanes arbustives à Detarium microcarpum. Ces savanes, très familières dans les paysages végétaux pâturés de la région, sont considérées comme un faciès de dégradation des formations originelles de la région. L'étude met en évidence une augmentation de la biodiversité avec I'augmentation de l'anthropisation et de la pression pastorale. Cette biodiversité élevée s'accompagne toutefois d'une dégradation de la valeur pastorale de la végétation. La production de biomasse et la valeur pastorale baissent de plus de la moitié en cas de pression pastorale forte. La part consommable de la biomasse est également très affectée car celle des espèces considérées comme fourragères médiocres atteint 34 à 68 p. 100 de la biomasse évaluée.
\end{abstract}

\section{INTRODUCTION}

La zone cotonnière du Burkina Faso et également, il semblerait, la sous-région où elle se trouve sont confrontées à une réduction des parcours naturels, consécutive à une augmentation rapide des mises en culture. Ces régions connaissent également une forte augmentation des effectifs de cheptel bovin à la suite de la sédentarisation des pasteurs et du développement de l'élevage chez les agriculteurs $(20,21,22,26)$. Il en résulte une double pression agricole et pastorale sur les terres. En effet, l'augmentation de l'emprise agricole par la mise en culture des savanes jusque-là inexploitées pose avec acuité la question de la gestion durable des terres et d'une exploitation raisonnée de la biodiversité (1). Le concept englobe différentes échelles biologiques (du gène à l'écosystème, en passant par l'espèce), spatiales, et des dimensions écologiques fonctionnelles et structurelles $(6,28)$. De manière plus pragmatique, la biodiversité mesure l'hétérogénéité globale d'un ensemble

1. Inera Farako-Bâ, Bobo-Dioulasso, Burkina Faso.

2. Cirad, URP Pastoralisme, Montpellier, F-34000 France ;

Cnrs, Montpellier, F-34000 France.

3. Cirad, URP Pastoralisme, Montpellier, F-34000 France :

Cirdes, Bobo-Dioulasso, Burkina Faso.

*Auteur pour la correspondance

Inera Farako-Bâ, BP 910 Bobo-Dioulasso, Burkina Faso.

E-mail : edwigebot@hotmail.com dénombrable dont les éléments peuvent être regroupés en catégories, donc d'un ensemble partitionnable (29). Le maintien de cette biodiversité est au cœur des questions de développement durable. Ainsi, l'un des défis pour le pâturage des animaux domestiques est d'assurer le maintien de cette biodiversité et l'intégrité des paysages pastoraux (4). Toutefois, l'action anthropique et, en particulier, l'impact de la pâture sur la biodiversité végétale sont assez controversés $(2,14,18,27)$. Ils sont tantôt considérés comme un facteur favorable à l'augmentation de la biodiversité, tantôt comme un facteur d'homogénéisation de la flore et des paysages. Les auteurs qui ont abordé la question recommandent la prudence, car l'impact du pâturage est à moduler en fonction de plusieurs paramètres : la zone agroclimatique, la pression anthropique globale, l'intensité du pâturage, la saison de pâture, etc. $(8,9)$.

L'objectif de la présente étude a été de comprendre et d'analyser le mécanisme d'évolution des végétations pâturées dans le contexte de forte pression foncière et pastorale qui prévaut dans les zones cotonnières de l'ouest du Burkina Faso, et d'en déduire les conséquences pour l'élevage dont l'alimentation est encore largement dépendante des parcours communautaires. L'accent a été porté sur la diversité spécifique à l'échelle des communautés végétales $(6$, 7,10 ) et tout particulièrement sur l'analyse de la strate herbacée qui fournit l'essentiel de l'alimentation des ruminants domestiques dans cette région. Les herbacées ont également une valeur indicatrice plus pertinente que les ligneux pour révéler les déséquilibres provoqués par l'action humaine (9). 
MATERIEL ET METHODES

\section{Sites d'étude}

L'étude a été menée dans trois terroirs présentant un gradient de pression foncière et pastorale. Ils se situent en zone sud-soudanienne à l'ouest du Burkina Faso. C'est la partie la plus arrosée du pays avec des précipitations qui varient de 900 à $1200 \mathrm{~mm}$ de pluie. La végétation actuelle se compose essentiellement de formations savanicoles à Vitellaria paradoxa et andropogonées vivaces. Elle est fortement modifiée par les activités anthropiques.

Le terroir d'Ouara, dans les anciens fronts pionniers de migration, est situé en pleine zone cotonnière. Avec un taux d'occupation agricole évalué à 47 p. 100 en 1998, ce terroir est représentatif des dynamiques agraires dans la zone cotonnière du Burkina Faso. Les pressions foncière et animale y sont fortes (tableau I). Les dynamiques agraires observées dans ce terroir se généralisent dans toute la région Ouest. Cette situation est consécutive au glissement des courants migratoires de populations et des aires d'élevage vers de nouveaux fronts pionniers qui se situent actuellement dans l'extrême sud-ouest du pays. A cet égard, ce terroir constitue un observatoire pour une analyse critique des interactions élevage et biodiversité. Les sols du terroir sont de type ferrugineux tropical lessivé induré. Dans l'ensemble, ce sont des sols peu profonds, la carapace apparaissant à 30-35 cm. Ils ont une texture sablo-limoneuse en surface $(0-12 / 19 \mathrm{~cm})(23)$.

Le terroir de Torokoro est représentatif des nouveaux fronts pionniers dans la partie méridionale du pays où convergent éleveurs et migrants agricoles à la recherche de terres et de pâturages. Le taux d'occupation agricole était encore faible dans l'ensemble : 17 p. 100 en 1998. La densité de population et du cheptel était plus faible qu'à Ouara (tableau I). Les stations étudiées se développent sur des sols à texture semblable à ceux d'Ouara mais localement plus profonds.

Le troisième site, la zone de pâture de Tierkoura, est exclusivement réservé à l'élevage. Jusqu'à une date récente, la forte infestation glossinaire interdisait son utilisation. Suite à des actions d'assainissement, son exploitation est réglementée depuis 2001. Le mode de gestion expérimenté prévoit un accueil de 90 p. 100 des animaux des terroirs riverains pendant la période agricole, soit du 15 mai au $1^{\mathrm{er}}$ février. De mai à novembre 2001, la zone avait accueilli environ 5100 bovins (soit une charge de 1,26 UBT/ha) *. Comme dans les deux autres sites, la station étudiée se trouve sur des sols peu profonds à texture sablo-limoneuse.

\section{Enquêtes phyto-écologiques}

L'impact des troupeaux domestiques sur la flore et la végétation des parcours communautaires est difficile à quantifier en condition réelle (multifonctionnalité des espaces, impossibilité de connaître les charges animales réelles à cause des fluctuations saisonnières des effectifs, etc.). A l'instar d'autres auteurs, on admet que les évolutions constatées à un moment donné sont la résultante de facteurs écologiques (sols, climat) et d'une action combinée de plusieurs facteurs anthropiques dont certains sont localement déterminants dans les processus d'évolution. Pour apprécier l'importance de la pression foncière, deux critères ont été considérés :

- la densité de population; elle était relativement plus forte à Ouara (57 habitants $/ \mathrm{km}^{2}$ ) par rapport à Torokoro (11 habitants/ $\mathrm{km}^{2}$ ) ; dans la zone de pâture de Tierkoura, elle ne faisait l'objet d'aucune occupation humaine à l'exception, temporaire, des pasteurs ;

* UBT : unité bovin tropical, un animal adulte pesant $250 \mathrm{~kg}$; bovin $=0,75 \mathrm{UBT}$; caprin et ovin $=0,1 \mathrm{UBT}$.

\section{Tableau}

Quelques caractéristiques des sites

\begin{tabular}{lccc} 
& $\begin{array}{c}\text { Terroir } \\
\text { d'Ouara }^{\prime}\end{array}$ & $\begin{array}{c}\text { Terroir } \\
\text { de Torokoro }\end{array}$ & $\begin{array}{c}\text { Zone pastorale } \\
\text { de Tierkoura }\end{array}$ \\
\hline $\begin{array}{l}\text { Superficie } \\
\text { (en hectares) }\end{array}$ & 7966 & 16190 & 4000 \\
$\begin{array}{l}\text { Taux d'occupation } \\
\text { agricole }\end{array}$ & $47 \%$ & $17 \%$ & 0 \\
$\begin{array}{l}\text { Densité de population } \\
\text { (habitants/km }{ }^{2} \text { ) }\end{array}$ & 57 & 11 & 0 \\
$\begin{array}{l}\text { Charge animale } \\
\text { (UBT *) }\end{array}$ & 2361 & 1467 & 3825 \\
$\begin{array}{l}\text { Densité animale } \\
\text { UBT/ha }\end{array}$ & 0,31 & 0,09 & 1,26 \\
\hline
\end{tabular}

$* 1$ bovin $=0,75 \mathrm{UBT} ; 1$ ovin ou un caprin $=0,1 \mathrm{UBT}$

- le taux d'occupation agricole; il était important à Ouara (47 p. 100), faible à Torokoro (17 p. 100) et nul dans la zone de pâture qui ne faisait l'objet d'aucune mise en culture.

L'intensité de la pression de pâturage a été appréciée sur la base de deux critères :

- la charge animale présente; elle était élevée à Ouara (0,31 UBT/ha), faible à Torokoro $(0,09 \mathrm{UBT} / \mathrm{ha})$, élevée mais ponctuelle dans la zone de pâture de Tierkoura $(1,26 \mathrm{UBT} / \mathrm{ha})$;

- l'ancienneté de l'utilisation pastorale ; l'augmentation des effectifs de cheptels, consécutive à l'installation des éleveurs à Ouara, date du milieu des années 1970, alors qu'elle est récente pour Torokoro (milieu des années 1995). La zone de pâture de Tierkoura n'a été réellement exploitée qu'à partir de 2001.

Des critères précédents, trois niveaux de perturbation ont été déduits :

- le niveau 1 , où la pression foncière a été nulle et la pression pastorale élevée mais ponctuelle; la pression anthropique globale sur les pâturages a été considérée comme faible ; c'était la situation de la zone pastorale de Tierkoura exclusivement réservée au pâturage ;

- le niveau 2, où la pression foncière a été faible associée à une pression pastorale modérée; la pression anthropique globale sur les pâturages a été considérée comme modérée ; c'était la situation du terroir de Torokoro ;

- le niveau 3 , où la pression foncière a été forte associée à une pression pastorale également forte ; la pression anthropique globale sur les pâturages a été considérée comme forte ; le terroir d'Ouara était représentatif de cette situation.

Selon une approche synchronique, des faciès de savanes arbustives à Detarium microcarpum ont été étudiés. D. microcarpum est un arbuste classé comme plante envahissante à l'origine de l'embroussaillement des pâturages soudaniens (3). Ces savanes sont considérées comme un faciès de dégradation des formations originales de la région. Elles sont très familières dans les paysages végétaux pâturés et existent un peu partout à l'état de jachère ou de formations naturelles. Elles sont régulièrement parcourues par les feux de brousse qui sont un facteur anthropique commun à toutes ces savanes. Les termes de formations naturelles sont abusivement utilisés, car les phytogéographes qui ont étudié les savanes africaines argumentent qu'elles ont été, à un moment donné de leur existence, exploitées par l'homme. Dans le cas présent, ces termes sont utilisés pour désigner toutes les formations qui n'ont subi que 
des perturbations mineures, en particulier celles qui n'ont pas fait l'objet de défrichement de mémoire d'homme.

Les relevés de végétation ont été effectués de la mi-septembre à début novembre 2002. Six stations de $2500 \mathrm{~m}^{2}$ chacune $(50 \mathrm{~m} \mathrm{x}$ $50 \mathrm{~m}$ ) ont été étudiées : deux stations de savanes naturelles, dont l'une dans la zone de pâture de Tierkoura (savane naturelle 1) et l'autre à Torokoro (savane naturelle 2), et quatre anciennes jachères de plus de 15 ans, dont l'une à Torokoro (jachère 1) et les trois autres à Ouara (jachère 2 , jachère 3 et jachère 4). Le tableau II donne la répartition de ces stations en fonction de l'intensité de la pression foncière et pastorale. Dans chacune des stations, une placette de $16 \mathrm{~m}^{2}$ (4 m x $4 \mathrm{~m}$ ) a été mise en défens pour l'étude de la strate herbacée. La taille des placettes a été choisie en fonction de l'aire minimale pour la strate ligneuse et herbacée dans les savanes soudaniennes $(30,33)$.

La composition floristique et l'organisation spatiale du tapis herbacé ont été étudiées par la méthode des points quadrats (11). La liste floristique a été complétée par l'inventaire des espèces non recensées sur la ligne de lecture. Chaque placette a été ensuite fauchée et triée en deux catégories fourragères : les graminées (vivaces et annuelles) et les autres espèces (herbacées diverses et cypéracées). La biomasse a été séchée à l'air libre, puis à l'étuve pour la détermination du taux de matière sèche (MS). Quant à la strate ligneuse, des comptages ont été effectués en fonction des strates: la strate des buissons, des régénérations et des petits arbustes

\section{Tableau II}

Répartition des stations en fonction de l'intensité de la pression foncière et de pâturage

\begin{tabular}{lccc} 
& \multicolumn{3}{c}{ Pression anthropique globale } \\
\cline { 2 - 4 } & Niveau 1 & Niveau 2 & Niveau 3 \\
Savanes naturelles & 1 & 1 & 0 \\
Jachères & 0 & 1 & 3 \\
Total stations & 1 & 2 & 3
\end{tabular}

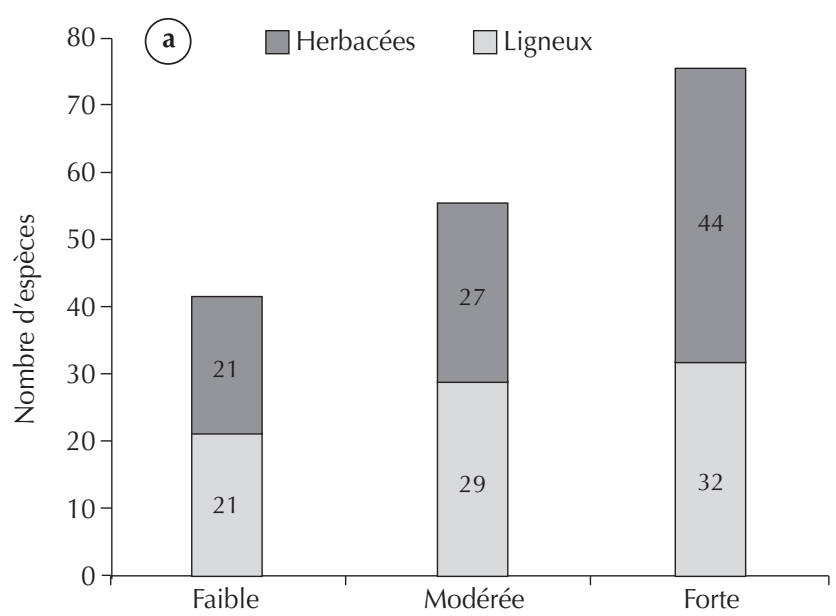

(moins de $2 \mathrm{~m}$ de hauteur), la strate des arbustes et des jeunes arbres (2-5 m), et la strate des arbres (plus de $5 \mathrm{~m}$ de hauteur).

La biodiversité de la flore a été approchée à partir de la richesse floristique. La diversité- $\alpha$ correspondait au nombre d'espèces inventoriées dans une station donnée $(6,7,32)$. La comparaison des flores interstationnelle (diversité- $\gamma$ ) a été calculée grâce à la distance de Hamming (10). Elle s'écrit $\mathrm{H}=1-\mathrm{J}$ où $\mathrm{J}$ est le coefficient de communauté de Jaccard (24) égal au rapport du nombre d'espèces communes entre deux relevés.

On retient les échelles de valeurs suivantes : différence floristique très faible pour $\mathrm{H}<20$; faible pour $20 \leq \mathrm{H}<40$; moyenne pour $40 \leq \mathrm{H}<60$; forte pour $60 \leq \mathrm{H}<80$; très forte pour $80 \leq \mathrm{H}$.

La diversité de la végétation a été calculée à l'aide de l'indice de Shannon (31) :

$\mathrm{H}=-\sum\left(\mathrm{Pi} \log _{2} \mathrm{Pi}\right)$

avec $\mathrm{Pi}$ = probabilité de rencontrer l'espèce $\mathrm{i}$ et exprimé par la contribution spécifique de l'espèce. Cet indice varie de zéro (une seule espèce) à $\log _{2} \mathrm{~N}$ (lorsque toutes les espèces ont la même abondance). De façon générale, plus il y a d'espèces avec une abondance voisine, plus la diversité est élevée.

\section{RESULTATS}

\section{Impact du pâturage sur la richesse floristique et le spectre fourrager}

Les stations les plus anthropisées et qui subissaient en outre une forte pression pastorale (niveau 3), en l'occurrence les jachères anciennes d'Ouara, avaient la plus grande richesse floristique. En moyenne 76 espèces ont été recensées à Ouara contre 56 à Torokoro et 42 en zone pastorale (figure 1a). La différence floristique entre stations a surtout été remarquable au niveau de la flore herbacée.

Quatre catégories d'espèces ont été retenues pour caractériser le spectre fourrager et rendre compte de la répartition des espèces en fonction de leur intérêt fourrager : (i) les graminées vivaces qui, en dehors de conditions édaphiques particulières, dominent dans les savanes sud-soudanienne en équilibre; (ii) les graminées annuelles qui s'installent dans les premiers stades postculturaux ou dans des conditions édaphiques particulières ; (iii) les herbacées

Intensité de la pression pastorale

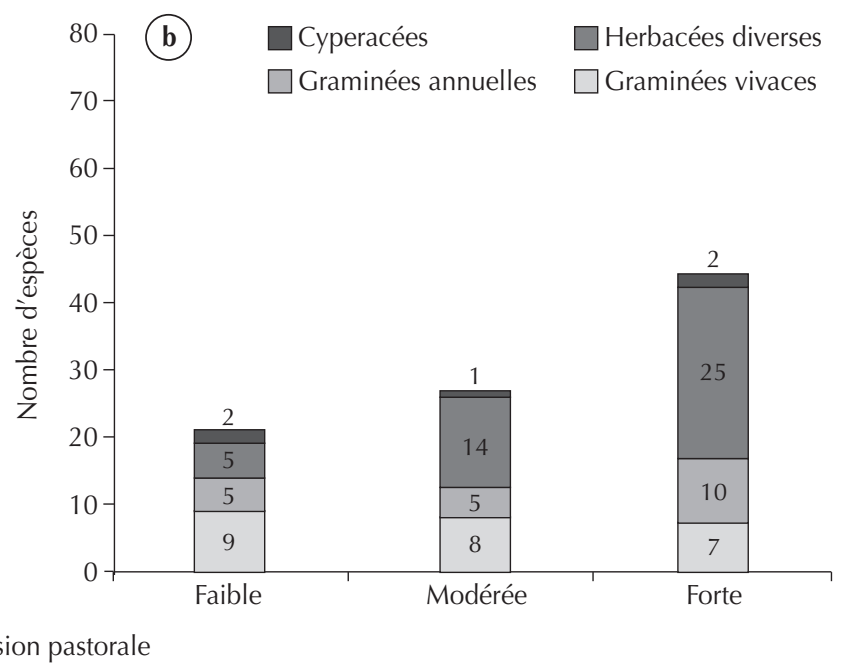

Figure 1 : richesse floristique et spectre fourrager de quelques faciès de savanes arbustives à Detarium microcarpum. 
diverses qui regroupent différentes dicotylédones d'intérêt pastoral faible à nul ; (iv) les cypéracées qui ont également un intérêt pastoral faible.

Dans toutes les stations, se retrouvait un fond d'espèces communes composé de quelques graminées vivaces de savanes (Andropogon ascinodis, Schizachyrium sanguineum, Monocymbium ceresiiforme), auxquelles s'ajoutait un nombre important d'herbacées diverses et de graminées annuelles. Toutefois, il y avait peu d'espèces communes aux stations à faible pression pastorale et à celles subissant une pression pastorale modérée à forte. La différence entre les flores des différentes stations calculée par la distance de Hamming a été ainsi très forte entre les stations subissant une pression pastorale faible et les autres $(\mathrm{H}>80)$. Elle a été forte à très forte entre les stations subissant une pression pastorale modérée et forte, selon qu'il s'agissait de formation naturelle ou de jachère (tableau III).

Le spectre fourrager a révélé (figure 1b) que c'étaient les espèces de la catégorie herbacées diverses qui faisaient la différence entre stations. La proportion de cette catégorie a augmenté avec l'intensité de la pression pastorale. La richesse de la flore dans cette catégorie dans les stations à forte pression pastorale a révélé un premier signe de dégradation de la flore pastorale par l'éviction des graminées vivaces. Parmi ces herbacées diverses ont été recensées de nombreuses dicotylédones dont certaines étaient également indicatrices d'épuisement des parcours (Spermacoce spp., Indigofera spp., Tephrosia spp., etc.), ainsi que des espèces postculturales.

\section{Impact du pâturage sur la structure de la strate herbacée}

\section{Recouvrement}

Les contributions spécifiques cumulées pour chaque catégorie d'herbacées (graminées vivaces, graminées annuelles, herbacées diverses et cypéracées), présentées dans la figure 2, font ressortir que, lorsque la pression pastorale était peu importante (niveau 1), le sol était presque entièrement recouvert par les graminées vivaces (99 p. 100). Les espèces Andropogon ascinodis et Hyparrhenia spp. étaient les plus abondantes et participaient pour 89 à 99 p. 100 au recouvrement total. En revanche, avec l'augmentation de la

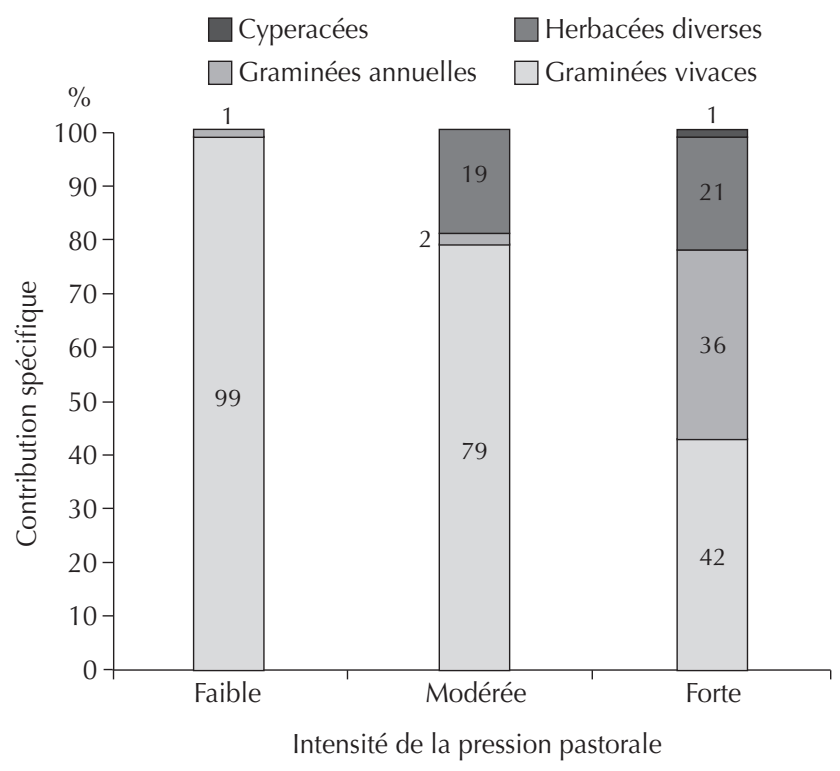

Figure 2 : importance des types biologiques dans la végétation herbacée.

pression pastorale (niveaux 2 et 3), la part de ces graminées vivaces dans le recouvrement était moindre. Entre les niveaux d'intensité de pâturage 2 et 3 , la participation des graminées annuelles, respectivement de 2 et 36 p. 100, faisait la différence. Cette catégorie a pris de plus en plus d'importance avec l'intensité de pâturage.

\section{Diversité informatique de Shannon}

L'indice de diversité de Shannon prend en compte non seulement la richesse floristique mais également la proportion de chaque espèce au sein du tapis herbacé. Il a été calculé à partir des fréquences centésimales (Fci) des espèces. La tendance au niveau des jachères a été la suivante: plus la pression pastorale était forte, plus la diversité était élevée (figure 3). Cette situation peut être interprétée comme la conséquence d'un habitat où de nombreuses espèces ont pu s'installer du fait de la perturbation créée par la pâture.

Tableau III

Différence entre flore herbacée calculée par la distance de Hamming

\begin{tabular}{|c|c|c|c|c|c|c|c|}
\hline & & \multicolumn{6}{|c|}{ Pression pastorale } \\
\hline & & \multirow{2}{*}{$\begin{array}{c}\text { Niveau } 1 \\
\text { Savane } \\
\text { naturelle } 1\end{array}$} & \multicolumn{2}{|c|}{ Niveau 2} & \multicolumn{3}{|c|}{ Niveau 3} \\
\hline & & & $\begin{array}{c}\text { Savane } \\
\text { naturelle } 2\end{array}$ & Jachère 1 & Jachère 2 & Jachère 3 & Jachère 4 \\
\hline Niveau 1 & Savane naturelle 1 & 0 & & & & & \\
\hline \multirow[t]{2}{*}{ Niveau 2} & Savane naturelle 2 & $* * *$ & 0 & & & & \\
\hline & Jachère 1 & $* * *$ & $* *$ & 0 & & & \\
\hline \multirow[t]{3}{*}{ Niveau 3} & Jachère 2 & $* * *$ & $* * *$ & $* *$ & 0 & & \\
\hline & Jachère 3 & $* * *$ & $* * *$ & $* *$ & $*$ & 0 & \\
\hline & Jachère 4 & $* * *$ & $* * *$ & $* *$ & $*$ & $*$ & 0 \\
\hline
\end{tabular}




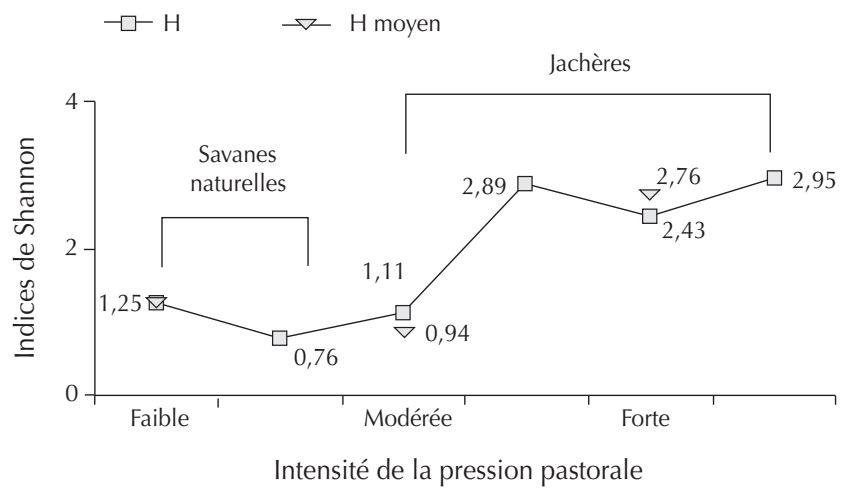

Figure 3 : évolution de l'indice de diversité de Shannon en fonction de la pression de pâturage d'après la distance de Hamming (cité dans Daget, 2003).

En revanche, pour les formations naturelles, une tendance inverse a été observée. Les auteurs ont calculé des indices de 1,25 et 0,76 respectivement pour la station subissant une pression pastorale faible et celle subissant une pression modérée. Dans cette dernière, la dominance marquée au profit de deux espèces (Andropogon ascinodis et Hyparrhenia subplumosa) expliquait la faible diversité du tapis végétal. En effet, les deux espèces à elles seules contribuaient pour 93 p. 100 au recouvrement herbacé. Cette forte dominance pouvait être mise en relation avec l'effet bénéfique du broutage en cas de pression pastorale modérée qui favorisait le tallage des meilleures espèces sélectionnées par le bétail. Une forte dominance de quelques espèces entraîne une baisse de la diversité de la végétation. La probabilité de recenser les mêmes espèces à chaque point de lecture était forte. En revanche, l'équirépartition des individus dans la station à faible pression de pâturage indique une diversité plus élevée. En effet, quatre espèces ont été interceptées sur la ligne de lecture : Andropogon ascinodis, Hyparrhenia subplumosa, Urelytrum sp. et Schizachyrium sanguineum, avec respectivement 48, 26, 16 et 10 p. 100 du recouvrement. La probabilité de recenser les mêmes espèces à chaque point de lecture était plus faible.

\section{Biodiversité et potentiel fourrager}

\section{Impact du pâturage sur la productivité des parcours}

La production de biomasse dans la savane naturelle subissant une pression pastorale faible était plus forte que celle de son homologue en situation de pression pastorale modérée (figure 4) : respectivement 2,6 t MS/ha contre 1,9 $6 \mathrm{t} \mathrm{MS/ha.} \mathrm{Cette} \mathrm{différence} \mathrm{était}$ attribuée aux effets du pâturage, cause de la différence floristique très forte entre les deux stations (tableau III).

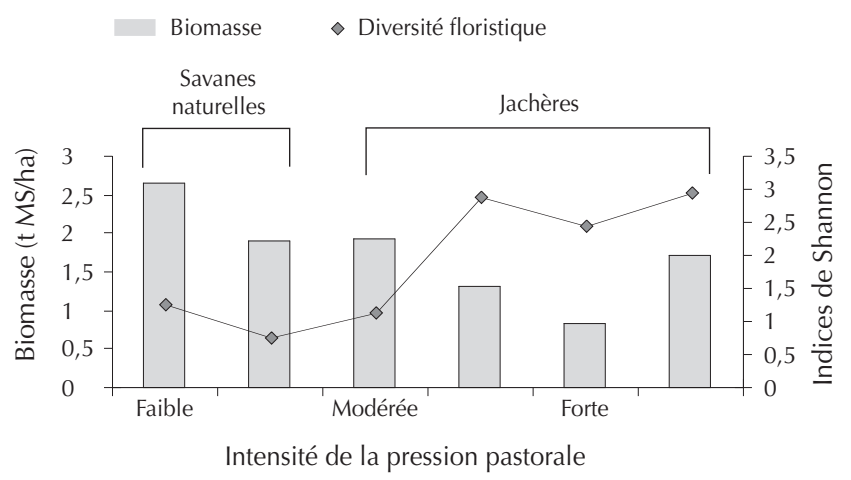

Figure 4 : évolution de la production des parcours en fonction de la diversité spécifique.
La production de biomasse des quatre stations de jachère a été en moyenne plus faible que celle des savanes naturelles. Les stations en situation de forte pression pastorale et anthropique (niveau 3), avec la diversité floristique la plus élevée, ont montré les plus faibles niveaux de production. La production moyenne de ces jachères a été évaluée à 1,2 t MS/ha contre 1,9 t MS/ha en pression pastorale modérée et 2,64 t MS/ha en situation de pression pastorale faible. Dans les stations subissant une forte pression pastorale, la part des herbacées diverses a représenté 26,71 à 48,39 p. 100 de la biomasse totale mesurée.

\section{Impact du pâturage sur la valeur pastorale}

La valeur pastorale $(12,13,15)$ est un indice bromatologique des herbages qui fait intervenir la composition floristique à travers la contribution spécifique présence (CPS) et la qualité fourragère des espèces ou indice spécifique. Elle permet une comparaison des herbages entre eux (33). Les valeurs moyennes ont été de 60, 49 et 35 p. 100 respectivement pour les pressions pastorales de niveaux 1 , 2 et 3 (figure 5). Les savanes naturelles, où les graminées vivaces, espèces les plus productives, ont contribué fortement au recouvrement herbacé, ont montré aussi les valeurs pastorales les plus élevées. D'une façon générale, les résultats ont montré que la valeur pastorale des pâturages baissait avec l'augmentation de la pression pastorale, à cause de la diminution de la contribution des bonnes espèces fourragères.

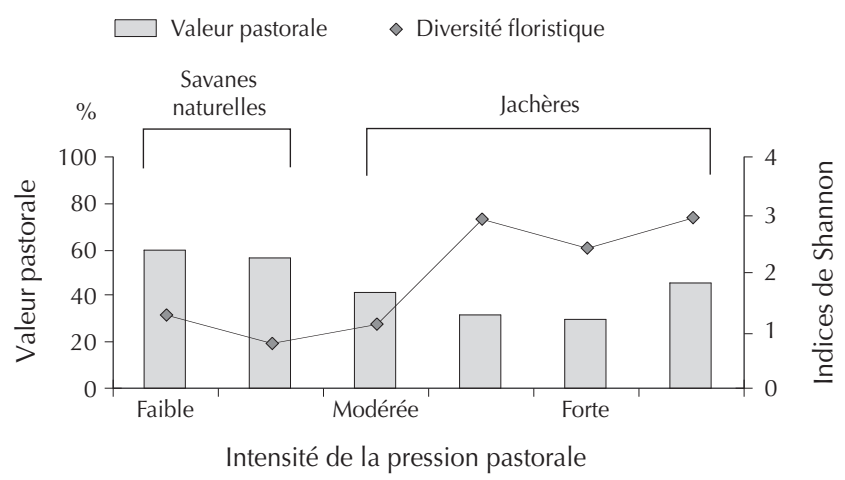

Figure 5 : évolution de la production des parcours en fonction de la diversité spécifique.

\section{DISCUSSION}

La qualité des pâturages sud-soudaniens dépend avant tout de la proportion des graminées vivaces, les espèces les plus productives et les plus consommées par le bétail $(9,16)$. En dehors des situations édaphiques particulières, le tapis herbacé des savanes soudanaises en équilibre est constitué en grande majorité de graminées cespiteuses qui développent de larges touffes. Sur toute la gamme des sols ferrugineux tropicaux ou ferrallitiques, on retrouve à peu près les mêmes espèces ; seule leur proportion peut varier d'une savane à une autre (9). Ainsi les graminées vivaces, comme Andropogon ascinodis, Hyparrhenia subplumosa, Hyparrhenia smithiana, Schizachyrium sanguineum, ont été recensées dans plus de 70 p. 100 des relevés dans les stations peu perturbées. Quand ces formations sont mises en culture, une phase de jachère dont la durée est fonction de la pression foncière permet une reconstitution de la végétation.

En l'absence de perturbations majeures, de nombreux travaux attestent qu'au bout de 10 à 15 ans, les adventices des cultures et les plantes de jachères sont remplacées par la graminée vivace 
Andropogon gayanus qui finit toujours par disparaître au profit de graminées savanicoles, comme Andropogon ascinodis, Hyparrhenia spp., etc. $(9,17,33)$. L'importance des espèces annuelles dans ces savanes et dans les jachères anciennes, en particulier les dicotylédones classées dans la catégorie herbacées diverses et les graminées annuelles, est un signe de déséquilibre, défavorable aux graminées vivaces, entraînant donc une dégradation de la végétation pastorale.

Dans la présente étude de cas sur les savanes arbustives à Detarium microcarpum, l'importance de ces deux catégories d'espèces (herbacées diverses et graminées annuelles) dans la flore et la végétation a été utilisée comme indicateur d'évolution pour comparer différents faciès soumis à des pressions foncières et pastorales différentielles. L'examen de la flore en fonction de l'importance de la pression anthropique et pastorale a fait ressortir les faits majeurs suivants :

- une richesse floristique plus élevée dans les stations de jachère que dans celles de savanes naturelles. Cette situation résultait surtout de l'héritage des cycles culturaux précédents. En effet, de nombreuses espèces postculturales pouvaient se maintenir sous l'effet perturbateur du pâturage ;

- une augmentation de la richesse floristique avec l'augmentation de la pression pastorale. Cette augmentation a été surtout remarquable au niveau de la strate herbacée avec 44 espèces inventoriées en situation de forte pression pastorale contre 21 dans les stations moins perturbées. En effet, de nombreux travaux au Sahel et dans les savanes soudaniennes concluent à un maintien ou une augmentation de la richesse floristique herbacée en cas d'exploitation pastorale importante $(2,9,17,18,25)$;

- un nombre élevé d'espèces appartenant à la catégorie des herbacées diverses, en situation de pression pastorale modérée à forte. La proportion de cette catégorie d'espèces a augmenté avec l'intensité de la pression de pâturage. Elle a représenté respectivement 51 et 56 p. 100 du nombre d'espèces recensées en cas de pression pastorale modérée à forte, alors que leur proportion dans les savanes naturelles peu perturbées n'était que de 24 p. 100. Des valeurs similaires ont été trouvées par Hoffman (19) qui a analysé la flore des jachères pâturées en pays Lobi au nord-est de la Côte d'Ivoire. L'auteur évalue la proportion des herbacées diverses à 50,5 p. 100, alors qu'elle est comprise entre 3 et 14 p. 100 dans les savanes voisines moins perturbées. Selon César (9), la présence de nombreuses espèces de légumineuses (Tephrosia spp., Indigofera spp.) est un indicateur d'épuisement des sols, en l'occurrence une carence en azote ;

- une proportion de graminées annuelles variant entre 21 et 45 p. 100 des espèces inventoriées dans les stations subissant une forte pression pastorale. La présence d'espèces postculturales, comme Digitaria horizontalis, Eragrostis spp., a été enregistrée. Cette situation est révélatrice d'un processus d'évolution bloqué pour ces jachères anciennes où cette catégorie d'espèce devrait en principe avoir disparu. En effet, ces espèces qui sont des adventices des cultures ne subsistent que peu de temps après l'abandon de la parcelle. Lorsque ces espèces se maintiennent longtemps après l'abandon de la culture, cela indique un fort épuisement du sol ou une dégradation par le pâturage (9) ;

- des espèces indicatrices de mauvaise gestion pastorale, voire de surpâturage, ont été recensées dans les stations subissant une forte pression pastorale. Il s'agissait d'espèces classées dans la catégorie des refus, comme Elionurus pobeguinii, Panicum phragmitoides, qui se multipliaient au détriment des espèces bonnes fourragères, lesquelles, au contraire, avaient tendance à régresser sous l'effet de la sélection par le bétail.
L'analyse de la structure de la végétation a également révélé l'importance des graminées annuelles et des herbacées diverses, constatée au niveau de la flore des stations subissant une forte pression pastorale (niveau 3), où la contribution au recouvrement des deux catégories a varié de 48 à 63 p. 100. La graminée annuelle Digitaria horizontalis a, par exemple, contribué à 30 p. 100 du recouvrement dans certaines stations. Dans les stations subissant une pression pastorale modérée (niveau 2), la contribution des herbacées diverses et des graminées annuelles a été évaluée à 38 p. 100 du recouvrement. Toutefois, les espèces Elionurus pobeguinii, Panicum phragmitoides, Spermacoce spp., etc., considérées comme indicatrices de surpâturage, n'ont pas été trouvées.

En revanche, l'analyse de la végétation n'a pas révélé la tendance à l'homogénéisation décrite au Sahel. En effet, des travaux en zone sahélienne du Niger montrent qu'en cas de pâturage important sur des jachères en saison de pluie, la végétation est dominée par une seule espèce ou par un petit nombre d'espèces qui représentent entre 50 et 90 p. 100 de la contribution spécifique (5). Sur des jachères surpâturées du Sud Niger, Hiernaux (18) rapporte que l'espèce Sida cordifolia fait à elle seule 46 à 74 p. 100 du couvert herbacé.

Quand on considère le niveau de production de biomasse et la qualité du pâturage dans les différentes situations de pressions foncières et d'intensité de pâturage, on met en évidence que l'activité humaine affecte négativement ces paramètres. En effet, les stations soumises à une pression pastorale forte (niveau 3) ont été celles dont la productivité et la valeur pastorale ont été les plus faibles. Sur les stations fortement pâturées, les espèces non ou peu consommables (essentiellement les légumineuses et autres dicotylédones diverses) ont constitué 27 à 48 p. 100 de la biomasse totale. Des situations similaires sont évoquées par Hiernaux (18) en zone sahélienne et soudano-sahélienne du Niger. Selon ces auteurs, les espèces non consommables représentent 53 à 61 p. 100 de la phytomasse des jachères surpâturées. Dans les savanes soudaniennes en équilibre, les légumineuses ne représentent jamais une masse importante, même si le nombre d'espèces est toujours assez important. A Nazinga, en zone sud-soudanienne du Burkina Faso, les légumineuses ne représentent que 4 p. 100 du biovolume (16). Selon le même auteur, elles ne contribueraient que pour 0,5 p. 100 de la biomasse dans les savanes guinéenne de la Côte d'Ivoire. Quant aux autres dicotylédones, en nombre d'espèces souvent important, leur biomasse est également faible et ne représente également que 4 p. 100 du biovolume à Nazinga. Pour ce qui concerne les monocotylédones diverses, dont différentes cypéracées, en particulier le genre Fimbristylis qui est le plus représenté à Ouara, leur biomasse est généralement insignifiante.

\section{CONCLUSION}

L'extension des zones cultivées au détriment des parcours naturels est un phénomène qui caractérise les dynamiques agraires dans de nombreux pays africains et pose le problème de la gestion durable de ces parcours. L'étude de cas sur un faciès des paysages végétaux les plus courants en zone sud-soudanienne du Burkina Faso permet d'émettre quelques hypothèses sur les interactions en cours, entre l'élevage et les milieux favorables à cette activité.

En comparant la flore et la végétation du terroir d'Ouara, représentatif des dynamiques agraires en cours dans cette région, avec d'autres végétations moins perturbées, on met en évidence que la pâture augmente la richesse floristique. La différence entre les flores des milieux moins perturbés et ceux soumis à une exploitation pastorale se fait surtout au niveau des herbacées diverses qui sont considérées comme des espèces fourragères médiocres à mauvaises. L'action du pâturage, en modifiant l'équilibre au niveau 
des graminées pérennes natives des savanes, ouvre le milieu à d'autres espèces appartenant à un cortège floristique différent. En revanche, l'augmentation de la biodiversité par l'action du pâturage détériore la valeur pastorale et la productivité des pâturages à cause de la régression des bonnes espèces fourragères au profit de plantes herbacées sans valeur pastorale. On peut donc conclure que l'augmentation de la pression foncière, consécutive à la réduction des espaces pâturables et à l'augmentation concomitante du cheptel sédentaire, entraîne une dégradation du potentiel fourrager des terroirs. En cas de forte pression anthropique, comme c'est le cas dans le terroir d'Ouara, le niveau de production de biomasse chute de plus de la moitié et, surtout, plus de la moitié de cette biomasse n'est pas consommée par le bétail. Au total, moins du quart de la biomasse disponible dans ce terroir est utilisable pour le bétail, et ce, sans tenir compte des pertes diverses occasionnées par le feu, les termites, le piétinement, etc. Le niveau de dégradation dans ce terroir a atteint un seuil où une simple diminution de la charge animale n'est plus suffisante pour régénérer les parcours. Il faudra y associer un éclaircissement de la strate arbustive par élimination des ligneux et une réintroduction de plantes fourragères.

\section{BIBLIOGRAPHIE}

1. ABBADIE L., 2000. Maîtrise des effets du changement global des savanes. Réseau GLOBALSAV ACI Ecologie quantitative. Paris, France, ministère délégué à l'Enseignement supérieur et à la Recherche, 8 p.

2. ACHARD F., HIERNAUX P., BANOIN M., 2001. Les jachères naturelles et améliorées en Afrique de l'Ouest. In : Floret C., Pontanier R., éds, De la jachère naturelle à la jachère améliorée. Le point des connaissances. Vol. 2. Montrouge, France, John Libbey Eurotext, p. 201-239.

3. AUDRU J., 1977. Les ligneux et sub-ligneux des parcours naturels soudano-guinéens en Côte d'Ivoire. Leur importance et les principes d'aménagement. Maisons Alfort, France, lemvt, 267 p.

4. BALENT G., ALARD D., BLANFORT V., GIBON A., 1998. Activités de pâturage, paysages et biodiversité. Ann. Zootech., 47 : 419-429.

5. BANOIN M., ACHARD F., 1998. Place de la jachère dans les systèmes d'élevage et comportement alimentaire du bétail sur un terroir du sudouest du Niger : Tiko. In : Floret C., Pontanier R., éds, La jachère et systèmes agraires, Niamey, Niger, 30 sept. - 02 oct. 1998. Paris, France, Orstom.

6. BARBAULT R., 1997. Biodiversité. Paris, France, Hachette, 160 p.

7. BLONDEL J., 1995. Biogéographie. Approche écologique et évolutive. Paris, France, Masson, 291 p.

8. BOUDET G., 1975. Manuel sur les pâturages tropicaux et les cultures fourragères. Paris, France, ministère de la Coopération, 254 p. (Manuel et précis d'élevage $n^{\circ} 4$ )

9. CESAR J., 1992. Etude de la production biologique des savanes de la Côte d'Ivoire et son utilisation par I'homme. Biomasse, valeur pastorale et production fourragère. Doct. Etat, université Pierre et Marie Curie, Paris, France, 672 p.

10. DAGET P., 2003. La biodiversité stationnelle et régionales : retour sur les concepts et les mesures. In : Coll. La biodiversité végétale, des plantes pour I'avenir, Association française pour la conservation des espèces végétales, bureau des ressources génétiques, Troyes, France, 13-15 nov. 2003.

11. DAGET P., POISSONET J., 1971. Une méthode d'analyse phytoécologique des prairies, critères d'application. Ann. agron., 22 : 5-41.

12. DAGET P., POISSONET J., 1972. Un procédé d'estimation de la valeur pastorale des pâturages. Fourrages, 49 : 31-39.

13. DAGET P., POISSONET J., 1990. Notion de valeur pastorale. Montpellier, France, Institut de Botanique. Repère, 3 : 5-8.

14. DAGET P., POISSONET J., 1997. Biodiversité et végétation pastorale, Revue Elev. Méd. vét. Pays trop., 50 : 141-144.

15. DELPECH R., 1960. Critères de jugement de la valeur agronomique des prairies. Fourrages, $4: 83-98$.

16. FOURNIER A., 1991. Phénologie, croissance et production végétales dans quelques savanes d'Afrique de l'Ouest. Variation selon un gradient climatique. Doct. Etat, université Pierre et Marie Curie, Paris, France, 312 p.

17. FOURNIER A., YONI M., ZOMBRE P., 2000. Les jachères à Andropogon gayanus en savane soudanienne dans l'ouest du Burkina Faso : flore, structure, déterminants et fonction dans l'écosystème. Etud. Flor. vég. Burkina Faso, 5 : 3-32.
18. HIERNAUX P., 1998. Effects of grazing on plant species composition and spatial distribution in rangelands of the Sahel. Plant Ecology, 33: 387-399.

19. HOFFMANN O., 1985. Pratiques pastorales et dynamique du couvert végétal en pays Lobi (nord-est de la Côte d'Ivoire). Paris, France, Orstom, $355 \mathrm{p}$.

20. INERA/CIRDES, 1997. Etude des systèmes d'élevage de la zone de Sidéradougou. Rapport de recherche. Bobo-Dioulasso, Burkina Faso, Inera/Cirdes, $60 \mathrm{p}$.

21. INERA/CIRDES, 1998. Etude des systèmes d'élevage de la partie nord de la zone agro-pastorale de Sidéradougou: Pratiques d'élevages intégrés aux exploitations agricoles et stratégies d'utilisation de l'espace. Rapport de recherche. Bobo-Dioulasso, Burkina Faso, Inera/Cirdes, 28 p.

22. INERA/GRN-SP, 1999. Historique dynamique des terroirs d'intervention du projet Front pionnier de migration. Rapport de recherche. Bobo-Dioulasso, Burkina Faso, Inera/GRN-SP.

23. INERA/GRN-SP-OUEST, 2000. Synthèse des activités du projet Fronts pionniers de migration. Rapport campagne 1998-99. Bobo-Dioulasso, Burkina Faso, Inera/GRN-SP.

24. JACCARD P., 1901. Lois de distribution de la flore alpine dans le bassin de Dranses et quelques régions voisines. Bull. Soc. vaudoise Sci. nat., $37: 241-272$.

25. KOITA B., BODIAN A., 2000. Evolution de la diversité végétale avec le temps de jachère en zone soudanienne au Sénégal. In : Floret $C$. Pontanier R., éds, La jachère en Afrique tropicale. Montrouge, France, John Libbey Eurotext, p. 408-414

26. LHOSTE P., 1988. Etude de l'élevage dans le développement des zones cotonnières. Maison-Alfort, France, Cirad-lemvt.

27. NOSBERGER J., MESSERLI M., CARLEN C., 1998. Biodiversity in grassland. Ann. Zootech., 47: 383-393.

28. NOSS R.F., 1990. Indicators for monitoring biodiversity: a hierarchical approach. Conserv. Biol., 4: 355-364.

29. POILECOT P., DAGET P., 2002. Contribution du service des herbiers du Cirad à l'étude de la biodiversité végétale. Montpellier, France, CiradEmvt, 16 p.

30. POISSONET J., CESAR J., 1972. Structure spécifique de la strate herbacée dans la savane à palmier rônier de Lamto (Côte d'Ivoire). Ann. Univ. Abidjan, E5 : 577-601.

31. SHANNON C., WEAWER W., 1948. The mathematical theory of communication. Urbana, IL, USA, Urbana University Press, p. 117-127.

32. WHITTAKER R., 1972. Evolution and measuremement of species diversity. Taxon, 21: 212-251.

33. ZOUNGRANA I., 1991. Recherche sur les aires pâturées au Burkina Faso. Doct. Etat, université de Bordeaux, France, 277 p.

Reçu le 05.09.2005, accepté le 04.07.2006 


\section{Summary}

Botoni/Liehoun E., Daget P., César J. Pasture Activities, Biodiversity and Pasture Vegetation in the Western Area of Burkina Faso

The biodiversity evolution of common access rangelands was studied based on three levels (high, medium, low) of land occupation and grazing pressure in the subhumid area of Burkina Faso. The study was carried out on shrubby savannahs of Detarium microcarpum. These grazed savannahs are very common in the region and are considered as a degraded pattern of the original vegetation. The study showed that vegetal diversity increased with human and grazing pressure. However, this high biodiversity led to the degradation of the shrubby rangeland feed value and of the vegetation in general. A high grazing pressure can reduce the biomass and pasture index by more than half. The palatable part of the biomass was also much affected, because that of species with low fodder value reached 34 to $68 \%$ of the measured biomass.

Keywords: Cattle - Biodiversity - Vegetation - Savanna Detarium microcarpum - Populations dynamics - Grazing Burkina Faso.

\section{Resumen}

Botoni/Liehoun E., Daget P., César J. Actividades de pastoreo, biodiversidad y vegetación pastoril en la zona oeste de Burkina Faso

La evolución de la biodiversidad vegetal basada en tres niveles de presión territorial y pastoril (débil, moderada, fuerte) ha sido estudiada en rutas comunitarias de la región subhúmeda de Burkina Faso. El estudio ha sido conducido en zonas de las sabanas de arbustos de Detarium microcarpum. Estas sabanas, muy familiares en los paisajes vegetales de pastoreo de la región, son consideradas como una faceta del deterioro de las formaciones originales de la región. El estudio pone en evidencia un aumento de la biodiversidad y un aumento de la intervención humana y de la presión pastoril. Esta elevada biodiversidad está siempre acompañada de un deterioro del valor pastoril de la vegetación. La producción de biomasa y el valor pastoril, bajan a más de la mitad en el caso de presión pastoril fuerte. La parte consumible de la biomasa se ve igualmente muy afectada ya que las especies consideradas como forraje mediocre alcanzan 34 a $68 \%$ de la biomasa evaluada.

Palabras clave: Ganado bovino - Biodiversidad - Vegetación Sabana - Detarium microcarpum - Dinámica de poblacións Pastoreo - Burkina Faso. 